\title{
Pullback attractors for asymptotically compact non-autonomous dynamical systems
}

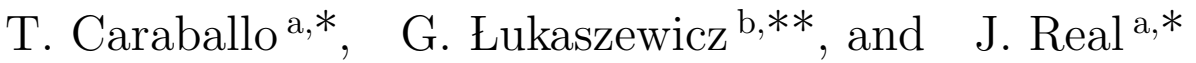 \\ ${ }^{a}$ Dpto. de Ecuaciones Diferenciales y Análisis Numérico, Universidad de Sevilla, \\ Apdo. de Correos 1160, 41080 Sevilla, Spain \\ ${ }^{\mathrm{b}}$ University of Warsaw, Institute of Applied Mathematics and Mechanics, \\ Banacha 2, 02-097 Warsaw, Poland
}

\begin{abstract}
First, we introduce the concept of pullback asymptotically compact non-autonomous dynamical system as an extension of the similar concept in the autonomous framework. Our definition is different from that of asymptotic compactness already used in the theory of random and non-autonomous dynamical systems (as developed by H. Crauel, F. Flandoli, P. Kloeden, B. Schmalfuss, amongst others) which means the existence of a (random or time-dependent) family of compact attracting sets. Next, we prove a result ensuring the existence of a pullback attractor for a nonautonomous dynamical system under the general assumptions of pullback asymptotic compactness and the existence of a pullback absorbing family of sets. This attractor is minimal and, in most practical applications, it is unique. Finally, we illustrate the theory with a 2D Navier-Stokes model in an unbounded domain.
\end{abstract}

Key words: non-autonomous (pullback) attractors, energy method, pullback asymptotically compact non-autonomous dynamical systems, cocycle, Navier-Stokes, unbounded domains Mathematics Subject Classifications (2000): 35B41, 35Q35

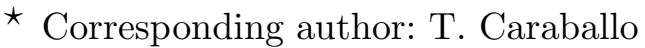

* Supported by Ministerio de Ciencia y Tecnología (Spain) and FEDER (European Community) grant BFM2002-03068

** Supported by Polish Government grant KBN 2 P303A 03022

Email addresses: caraball@us.es (T. Caraballo), glukasz@mimuw.edu.pl (G. Łukaszewicz), jreal@us.es (J. Real).
} 


\section{Introduction}

The understanding of the asymptotic behaviour of dynamical systems is one of the most important problems of modern mathematical physics. One way to treat this problem for a system having some dissipativity properties is to analyse the existence and structure of its global attractor, which in the autonomous case, is an invariant compact set which attracts all the trajectories of the system, uniformly on bounded sets. This set has, in general, a very complicated geometry which reflects the complexity of the long-time behaviour of the system (see, for instance, Cholewa \& Dlotko [6], Chueshov [7], Robinson [17], Temam [22], and the references therein).

However, non-autonomous systems are also of great importance and interest as they appear in many applications to natural sciences. On some occasions, some phenomena are modeled by nonlinear evolutionary equations which do not take into account all the relevant information of the real systems. Instead some neglected quantities can be modeled as an external force which in general becomes time-dependent (sometimes periodic, quasiperiodic or almost periodic due to seasonal regimes).

In the finite-dimensional framework (i.e. for non-autonomous ordinary differential equations in $\mathbb{R}^{N}$ ) the long-time behaviour of non-autonomous dynamical systems has been widely studied by means of the theory of skew-product flows (see the pioneering works by Miller [15], Sell [20]). However, most of the progress in the infinite-dimensional context, i.e., for non-autonomous partial differential equations and specially for systems appearing in mathematical physics, has been done during the last two decades.

The first attempts to extend the notion of global attractor to the non-autonomous case led to the concept of the so-called uniform attractor (see Chepyzhov and Vishik [5]). It is remarkable that the conditions ensuring the existence of the uniform attractor parallel those for autonomous systems. To this end, non-autonomous systems are lifted in [23] to autonomous ones by expanding the phase space. Then, the existence of uniform attractors relies on some compactness property of the solution operator associated to the system. However, one disadvantage of this uniform attractor is that it needs not be "invariant" unlike the global attractor for autonomous systems. At the same time, the theory of pullback (or cocycle) attractors has been developed for both the non-autonomous and random dynamical systems (see Crauel et al. [9], Langa and Schmalfuss [12], Kloeden and Schmalfuss [11], Schmalfuss [19]), and has shown to be very useful in the understanding of the dynamics of non-autonomous dynamical systems. In this case, the concept of pullback (or cocycle or non-autonomous) attractor provides a time-dependent family of compact sets which attracts families of sets in a certain universe (e.g. the bounded sets in the phase space) and satisfying an invariance property, what seems to be a natural set of conditions to be satisfied for an appropriate extension of the autonomous concept of attractor. Moreover, this cocycle formulation allows to handle more general time-dependent terms in the models not only the periodic, quasi periodic or almost periodic ones (see for instance Caraballo and Real [1], and Caraballo et al. [2] for non-autonomous models containing 
hereditary characteristics).

In order to prove the existence of the attractor (in both the autonomous and nonautonomous cases) the simplest, and therefore the strongest, assumption is the compactness of the solution operator associated with the system, which is usually available for parabolic systems in bounded domain. However, this kind of compactness does not hold in general for parabolic equations in unbounded domains and hyperbolic equations on either bounded or unbounded domain. Instead we often have some kind of asymptotic compactness. In this situation, there are several approaches to prove the existence of the global (or non-autonomous) attractor. Roughly speaking, the first one ensures the existence of the global (resp. non-autonomous) attractor whenever a compact attracting set (resp. a family of compact attracting sets) exists. The second method consists in decomposing the solution operator (resp. the cocycle or two-parameter semigroup) into two parts: a compact part and another one which decays to zero as time goes to infinity. However, as it is not always easy to find this decomposition, one can use a third approach which is based on the use of the energy equations which are in direct connection with the concept of asymptotic compactness. This third method has been used by Łukaszewicz and Sadowski in [14] (and later also in [16]) to extend to the non-autonomous situation the corresponding one in the autonomous framework (see Rosa [18]), but related to uniform asymptotic compactness. Our aim in this paper is to consider the case without uniformity properties and show how the pullback theory works in this situation. In this sense, it is worth mentioning that the compact case has been treated in Flandoli \& Schmalfuss [10] in the random case, so our results can be considered as natural complements (as it happens in the deterministic autonomous case).

The content of the paper is as follows. In Section 2 we introduce the concept of asymptotically compact non-autonomous dynamical system, and prove a general result ensuring the existence of a minimal pullback attractor under assumptions of asymptotic compactness and existence of a family of absorbing sets. Then, we apply our theory to prove the existence of a pullback attractor for a 2D Navier-Stokes model in an unbounded domain in which the external force needs not be bounded, neither almost periodic nor translation compact. It is enough that this term satisfies an appropriate integrability condition.

\section{Pullback attractors for asymptotically compact non-autonomous dynami- cal systems}

We suppose given a nonempty set $\Omega$ and a family $\left\{\theta_{t}\right\}_{t \in \mathbb{R}}$ of mappings $\theta_{t}: \Omega \rightarrow \Omega$ satisfying

i) $\theta_{0} \omega=\omega$ for all $\omega \in \Omega$,

ii) $\theta_{t}\left(\theta_{\tau} \omega\right)=\theta_{t+\tau} \omega$ for all $\omega \in \Omega, t, \tau \in \mathbb{R}$.

The operators $\theta_{t}$ are called the shift operators. 
Consider also a metric space $X$ with distance $\mathrm{d}(\cdot, \cdot)$, and a $\theta$-cocycle $\phi$ on $X$, i.e., a mapping $\phi: \mathbb{R}_{+} \times \Omega \times X \rightarrow X$, satisfying

a) $\phi(0, \omega, x)=x$ for all $(\omega, x) \in \Omega \times X$,

b) $\phi(t+\tau, \omega, x)=\phi\left(t, \theta_{\tau} \omega, \phi(\tau, \omega, x)\right)$, for all $t, \tau \in \mathbb{R}_{+},(\omega, x) \in \Omega \times X$.

The $\theta$-cocycle $\phi$ is said to be continuous if for all $(t, \omega) \in \mathbb{R}_{+} \times \Omega$, the mapping $\phi(t, \omega, \cdot)$ : $X \rightarrow X$ is continuous.

Let $\mathcal{P}(X)$ denote the family of all nonempty subsets of $X$, and $\mathcal{S}$ the class of all families $\widehat{D}=\{D(\omega) ; \omega \in \Omega\} \subset \mathcal{P}(X)$.

We consider given a nonempty subclass $\mathcal{D} \subset \mathcal{S}$.

Definition 1 The $\theta$-cocycle $\phi$ is said to be pullback $\mathcal{D}$-asymptotically compact $(\mathcal{D}$-a.c.) if for any $\omega \in \Omega$, any $\widehat{D} \in \mathcal{D}$, and any sequences $t_{n} \rightarrow+\infty, x_{n} \in D\left(\theta_{-t_{n}} \omega\right)$, the sequence $\phi\left(t_{n}, \theta_{-t_{n}} \omega, x_{n}\right)$ possesses a convergent subsequence.

Definition 2 A family $\widehat{B}=\{B(\omega) ; \omega \in \Omega\} \in \mathcal{S}$ is said to be pullback $\mathcal{D}$-absorbing if for each $\omega \in \Omega$ and $\widehat{D} \in \mathcal{D}$, there exists $t_{0}(\omega, \widehat{D}) \geq 0$ such that

$$
\phi\left(t, \theta_{-t} \omega, D\left(\theta_{-t} \omega\right)\right) \subset B(\omega) \quad \text { for all } t \geq t_{0}(\omega, \widehat{D}) .
$$

We denote by $\operatorname{dist}\left(C_{1}, C_{2}\right)$ the Hausdorff semi-distance between $C_{1}$ and $C_{2}$, defined as

$$
\operatorname{dist}\left(C_{1}, C_{2}\right)=\sup _{x \in C_{1}} \inf _{y \in C_{2}} d(x, y), \quad \text { for } \quad C_{1}, C_{2} \subset X \text {. }
$$

Definition 3 A family $\widehat{C}=\{C(\omega) ; \omega \in \Omega\} \in \mathcal{S}$ is said to be pullback $\mathcal{D}$-attracting if

$$
\lim _{t \rightarrow+\infty} \operatorname{dist}\left(\phi\left(t, \theta_{-t} \omega, D\left(\theta_{-t} \omega\right)\right), C(\omega)\right)=0 \quad \text { for all } \widehat{D} \in \mathcal{D}, \omega \in \Omega
$$

Definition 4 A family $\widehat{A}=\{A(\omega) ; \omega \in \Omega\} \in \mathcal{S}$ is said to be a global pullback $\mathcal{D}$-attractor if it satisfies

(1) $A(\omega)$ is compact for any $\omega \in \Omega$,

(2) $\widehat{A}$ is pullback $\mathcal{D}$-attracting,

(3) $\widehat{A}$ is invariant, i.e.

$$
\phi(t, \omega, A(\omega))=A\left(\theta_{t} \omega\right) \text { for any }(t, \omega) \in \mathbb{R}_{+} \times \Omega \text {. }
$$

Remark 5 Observe that Definition 4 does not guarantee the uniqueness of pullback $\mathcal{D}$ attractor (see Caraballo and Langa [3] for a discussion on this point).

Definition 6 For each $\widehat{D} \in \mathcal{S}$ and $\omega \in \Omega$, we define the omega-limit set of $\widehat{D}$ at $\omega$ (in 
the pullback sense) as

$$
\Lambda(\widehat{D}, \omega)=\bigcap_{s \geq 0}\left(\overline{\bigcup_{t \geq s} \phi\left(t, \theta_{-t} \omega, D\left(\theta_{-t} \omega\right)\right)}\right) .
$$

Obviously, $\Lambda(\widehat{D}, \omega)$ is a closed subset of $X$ that can be eventually empty. It is easy to see that for each $y \in X$, one has that $y \in \Lambda(\widehat{D}, \omega)$ if and only if there exist a sequence $t_{n} \rightarrow+\infty$ and a sequence $x_{n} \in D\left(\theta_{-t_{n}} \omega\right)$ such that

$$
\lim _{t_{n} \rightarrow+\infty} \mathrm{d}\left(\phi\left(t_{n}, \theta_{-t_{n}} \omega, x_{n}\right), y\right)=0 .
$$

Our first aim is to prove the following result on the existence of global pullback $\mathcal{D}$ attractor.

Theorem 7 Suppose the $\theta$-cocycle $\phi$ is continuous and pullback $\mathcal{D}$-asymptotically compact, and there exists $\widehat{B} \in \mathcal{D}$ which is pullback $\mathcal{D}$-absorbing. Then, the family $\widehat{A}$ defined by

$$
A(\omega)=\Lambda(\widehat{B}, \omega), \quad \omega \in \Omega,
$$

is a global pullback $\mathcal{D}$-attractor which is minimal in the sense that if $\widehat{C} \in \mathcal{S}$ is a family such that $C(\omega)$ is closed and $\lim _{t \rightarrow+\infty} \operatorname{dist}\left(\phi\left(t, \theta_{-t} \omega, B\left(\theta_{-t} \omega\right)\right), C(\omega)\right)=0$, then $A(\omega) \subset C(\omega)$.

Remark 8 First, notice that Theorem 7 generalises Theorem 3.5 in Flandoli 83 Schmalfuss [10].

On the other hand, if the universe $\mathcal{D}$ is inclusion closed (i.e., if $\widehat{D}^{\prime} \in \mathcal{S}, \widehat{D} \in \mathcal{D}$ and $D^{\prime}(\omega) \subset D(\omega)$ for all $\omega \in \Omega$, imply that $\left.\widehat{D}^{\prime} \in \mathcal{D}\right)$ and $\widehat{B} \in \mathcal{D}$ satisfies that $B(\omega)$ is closed for any $\omega \in \Omega$, then the assumptions in Theorem 7 imply that the ones in Theorem 1.8.1 from Chueshov [8] are also fulfilled. Conversely, given $\widehat{D} \in \mathcal{D}$ and $\varepsilon>0$, let us denote by $\widehat{D}_{\varepsilon}$ the family of subsets in $X$ formed by the $\varepsilon$-neighborhoods of $D(\omega)$ :

$$
D_{\varepsilon}(\omega)=\{x \in X: \quad \operatorname{dist}(x, D(\omega)) \leq \varepsilon\} .
$$

If we assume that $\mathcal{D}$ satisfies that for any $\widehat{D} \in \mathcal{D}$ there exists $\varepsilon>0$ such that $\widehat{D}_{\varepsilon} \in \mathcal{D}$ (what happens very often in applications, see for instance the example in Section 3), then the assumptions in Theorem 1.8.1 from Chueshov [8] also imply the ones in our Theorem 7.

Consequently, in this situation, we can claim that Theorem 1.8.1 in Chueshov [8] and Theorem 7 are equivalent, although it seems that our result fits better to analyse problems in unbounded domains.

Remark 9 If the universe $\mathcal{D}$ contains the families of fixed bounded sets (i.e. for any bounded $C \subset X$ it follows that $\widehat{C}=\{C(\omega) \equiv C, \omega \in \Omega\} \in \mathcal{D})$ then, Theorem 7 implies that, the global pullback $\mathcal{D}$-attractor $\widehat{A}$ (whose existence is guaranteed by this theorem) 
is formed by a family of compact sets which pullback attracts the bounded subsets of $X$, which implies the existence of the pullback attractor $\widehat{A}_{0}$ in the sense of Crauel et al. [9] (which is a family of compact sets, invariant and pullback attracting the bounded subsets of $X$ ), and is given by

$$
A_{0}(\omega)=\overline{\bigcup_{\substack{C \subset X \\ \text { bounded }}} \Lambda(C, \omega) .}
$$

Furthermore, by the minimality of $\widehat{A}_{0}$ it follows that $A_{0}(\omega) \subset A(\omega)$ for any $\omega \in \Omega^{1}$.

In order to prove Theorem 7, we first need the following results.

Proposition 10 If $\widehat{B} \in \mathcal{S}$ is a pullback $\mathcal{D}$-absorbing family, then

$$
\Lambda(\widehat{D}, \omega) \subset \Lambda(\widehat{B}, \omega) \text { for all } \widehat{D} \in \mathcal{D}, \omega \in \Omega \text {. }
$$

If in addition $\widehat{B} \in \mathcal{D}$, then

$$
\Lambda(\widehat{D}, \omega) \subset \Lambda(\widehat{B}, \omega) \subset \overline{B(\omega)} \text { for all } \widehat{D} \in \mathcal{D}, \omega \in \Omega
$$

Proof. Let us fix $\widehat{D} \in \mathcal{D}, \omega \in \Omega$, and $y \in \Lambda(\widehat{D}, \omega)$. There exist a sequence $t_{n} \rightarrow+\infty$, and a sequence $x_{n} \in D\left(\theta_{-t_{n}} \omega\right)$ such that

$$
\phi\left(t_{n}, \theta_{-t_{n}} \omega, x_{n}\right) \rightarrow y
$$

As $\widehat{B}$ is pullback $\mathcal{D}$-absorbing, for each integer $k \geq 1$ there exists a $t_{n_{k}} \in\left\{t_{n}\right\}$ such that $t_{n_{k}} \geq k$ and

$$
\phi\left(t_{n_{k}}-k, \theta_{-\left(t_{n_{k}}-k\right)}\left(\theta_{-k} \omega\right), D\left(\theta_{-\left(t_{n_{k}}-k\right)}\left(\theta_{-k} \omega\right)\right)\right) \subset B\left(\theta_{-k} \omega\right)
$$

In particular, as $x_{n_{k}} \in D\left(\theta_{-t_{n_{k}}} \omega\right)=D\left(\theta_{-\left(t_{n_{k}}-k\right)}\left(\theta_{-k} \omega\right)\right)$, we obtain from (6)

$$
y_{k}=\phi\left(t_{n_{k}}-k, \theta_{-t_{n_{k}}} \omega, x_{n_{k}}\right) \in B\left(\theta_{-k} \omega\right) \text {. }
$$

But then

$$
\begin{aligned}
\phi\left(t_{n_{k}}, \theta_{-t_{n_{k}}} \omega, x_{n_{k}}\right) & =\phi\left(\left(t_{n_{k}}-k\right)+k, \theta_{-t_{n_{k}}} \omega, x_{n_{k}}\right) \\
& =\phi\left(k, \theta_{-k} \omega, \phi\left(t_{n_{k}}-k, \theta_{-t_{n_{k}}} \omega, x_{n_{k}}\right)\right) \\
& =\phi\left(k, \theta_{-k} \omega, y_{k}\right)
\end{aligned}
$$

and consequently by $(5), \phi\left(k, \theta_{-k} \omega, y_{k}\right) \rightarrow y$, with $y_{k} \in B\left(\theta_{-k} \omega\right)$. Thus, $y$ belongs to $\Lambda(\widehat{B}, \omega)$.

Finally, observe that if $\widehat{y}$ belongs to $\Lambda(\widehat{B}, \omega)$, then there exist a sequence $\widehat{t}_{n} \rightarrow+\infty$ and a sequence $\widehat{x}_{n} \in B\left(\theta_{-\widehat{t}_{n}} \omega\right)$ such that $\phi\left(\widehat{t}_{n}, \theta_{-\widehat{t}_{n}} \omega, x_{n}\right) \rightarrow \widehat{y}$. But, as $\widehat{B}$ is pullback $\mathcal{D}$ absorbing, there exists $t_{0}(\widehat{B}, \omega) \geq 0$ such that $\phi\left(\widehat{t}_{n}, \theta_{-\widehat{t}_{n}} \omega, x_{n}\right) \in B(\omega)$ for all $\widehat{t}_{n} \geq t_{0}(\widehat{B}, \omega)$, and consequently $\widehat{y}$ belongs to $\overline{B(\omega)}$.

1 We thank Pedro Marín-Rubio for having pointed out this fact. 
Remark 11 As a straightforward consequence of Proposition 10, we have that if $\widehat{B} \in \mathcal{D}$ is pullback $\mathcal{D}$-absorbing, then

$$
\overline{\bigcup_{\widehat{D} \in \mathcal{D}} \Lambda(\widehat{D}, \omega)}=\Lambda(\widehat{B}, \omega)
$$

so that, under the assumptions in Theorem \%, we have

$$
A(\omega)=\Lambda(\widehat{B}, \omega)=\varlimsup_{\widehat{D} \in \mathcal{D}} \Lambda(\widehat{D}, \omega), \quad \omega \in \Omega .
$$

Proposition 12 If $\phi$ is pullback $\mathcal{D}$-asymptotically compact, then for $\widehat{D} \in \mathcal{D}$ and $\omega \in \Omega$, the set $\Lambda(\widehat{D}, \omega)$ is nonempty, compact, and

$$
\lim _{t \rightarrow+\infty} \operatorname{dist}\left(\phi\left(t, \theta_{-t} \omega, D\left(\theta_{-t} \omega\right)\right), \Lambda(\widehat{D}, \omega)\right)=0
$$

Proof. Let us fix $\widehat{D} \in \mathcal{D}$ and $\omega \in \Omega$. If we consider a sequence $t_{n} \rightarrow+\infty$, and a sequence $x_{n} \in D\left(\theta_{-t_{n}} \omega\right)$, then from the sequence $\phi\left(t_{n}, \theta_{-t_{n}} \omega, x_{n}\right)$ we can extract a convergent subsequence $\phi\left(t_{\mu}, \theta_{-t_{\mu}} \omega, x_{\mu}\right) \rightarrow y$. By its construction, $y$ belongs to $\Lambda(\widehat{D}, \omega)$, and thus this set is nonempty.

We know that $\Lambda(\widehat{D}, \omega)$ is closed, and consequently in order to prove its compactness it is enough to see that for any given sequence $\left\{y_{n}\right\} \subset \Lambda(\widehat{D}, \omega)$, we can extract a convergent subsequence. First, observe that as $y_{n} \in \Lambda(\widehat{D}, \omega)$, there exist $t_{n} \geq n$ and $x_{n} \in D\left(\theta_{-t_{n}} \omega\right)$ such that

$$
\mathrm{d}\left(\phi\left(t_{n}, \theta_{-t_{n}} \omega, x_{n}\right), y_{n}\right) \leq \frac{1}{n}
$$

As $\phi$ is pullback $\mathcal{D}$-a.c., from the sequence $\phi\left(t_{n}, \theta_{-t_{n}} \omega, x_{n}\right)$ we can extract a convergent subsequence $\phi\left(t_{\mu}, \theta_{-t_{\mu}} \omega, x_{\mu}\right) \rightarrow y$, and consequently, from (10), we also obtain $y_{\mu} \rightarrow y$.

Finally, if (9) does not hold, there exist $\varepsilon>0$, a sequence $t_{n} \rightarrow+\infty$, and a sequence $x_{n} \in D\left(\theta_{-t_{n}} \omega\right)$, such that

$$
\mathrm{d}\left(\phi\left(t_{n}, \theta_{-t_{n}} \omega, x_{n}\right), y\right) \geq \varepsilon \quad \text { for all } y \in \Lambda(\widehat{D}, \omega)
$$

But from the sequence $\phi\left(t_{n}, \theta_{-t_{n}} \omega, x_{n}\right)$ we can extract a convergent subsequence $\phi\left(t_{\mu}, \theta_{-t_{\mu}} \omega, x_{\mu}\right) \rightarrow y \in \Lambda(\widehat{D}, \omega)$, which contradicts $(11)$.

Proposition 13 If the $\theta$-cocycle $\phi$ is continuous and pullback $\mathcal{D}$-asymptotically compact, then for any $(t, \omega) \in \mathbb{R}_{+} \times \Omega$ and any $\widehat{D} \in \mathcal{D}$, one has

$$
\phi(t, \omega, \Lambda(\widehat{D}, \omega))=\Lambda\left(\widehat{D}, \theta_{t} \omega\right) .
$$

Proof. Let $(t, \omega) \in \mathbb{R}_{+} \times \Omega$ and $\widehat{D} \in \mathcal{D}$ be fixed, and $y \in \Lambda(\widehat{D}, \omega)$. We now show that $\phi(t, \omega, y) \in \Lambda\left(\widehat{D}, \theta_{t} \omega\right)$, and, consequently, we obtain that $\phi(t, \omega, \Lambda(\widehat{D}, \omega)) \subset \Lambda\left(\widehat{D}, \theta_{t} \omega\right)$. 
We already know that there exist a sequence $t_{n} \rightarrow+\infty$ and a sequence $x_{n} \in D\left(\theta_{-t_{n}} \omega\right)$ such that $\phi\left(t_{n}, \theta_{-t_{n}} \omega, x_{n}\right) \rightarrow y$. But $\phi\left(t, \omega, \phi\left(t_{n}, \theta_{-t_{n}} \omega, x_{n}\right)\right)=\phi\left(t+t_{n}, \theta_{-\left(t+t_{n}\right)}\left(\theta_{t} \omega\right), x_{n}\right)$, and, as $\phi$ is continuous, it follows that $\phi\left(t+t_{n}, \theta_{-\left(t+t_{n}\right)}\left(\theta_{t} \omega\right), x_{n}\right) \rightarrow \phi(t, \omega, y)$, with $t+t_{n} \rightarrow+\infty$, and $x_{n} \in D\left(\theta_{-t_{n}} \omega\right)=D\left(\theta_{-\left(t+t_{n}\right)}\left(\theta_{t} \omega\right)\right)$, and therefore $\phi(t, \omega, y) \in \Lambda\left(\widehat{D}, \theta_{t} \omega\right)$.

Now, we prove $\Lambda\left(\widehat{D}, \theta_{t} \omega\right) \subset \phi(t, \omega, \Lambda(\widehat{D}, \omega))$. For $y \in \Lambda\left(\widehat{D}, \theta_{t} \omega\right)$ there exist a sequence $t_{n} \rightarrow+\infty$ and a sequence $x_{n} \in D\left(\theta_{-t_{n}}\left(\theta_{t} \omega\right)\right)$ such that

$$
\phi\left(t_{n}, \theta_{-t_{n}}\left(\theta_{t} \omega\right), x_{n}\right) \rightarrow y
$$

If $t_{n} \geq t$, we have

$$
\begin{aligned}
\phi\left(t_{n}, \theta_{-t_{n}}\left(\theta_{t} \omega\right), x_{n}\right) & =\phi\left(t+\left(t_{n}-t\right), \theta_{-\left(t_{n}-t\right)} \omega, x_{n}\right) \\
& =\phi\left(t, \omega, \phi\left(t_{n}-t, \theta_{-\left(t_{n}-t\right)} \omega, x_{n}\right)\right) .
\end{aligned}
$$

But, as $\phi$ is pullback $\mathcal{D}$-a.c., $t_{n}-t \rightarrow+\infty$, and $x_{n} \in D\left(\theta_{-t_{n}}\left(\theta_{t} \omega\right)\right)=D\left(\theta_{-\left(t_{n}-t\right)} \omega\right)$, one can ensure that there exists a subsequence $\left\{\left(t_{\mu}, x_{\mu}\right)\right\} \subset\left\{\left(t_{n}, x_{n}\right)\right\}$ such that

$$
\phi\left(t_{\mu}-t, \theta_{-\left(t_{\mu}-t\right)} \omega, x_{\mu}\right) \rightarrow z \in \Lambda(\widehat{D}, \omega),
$$

and then, by (14) and (13), we have $y=\phi(t, \omega, z) \in \phi(t, \omega, \Lambda(\widehat{D}, \omega))$.

Now, we can prove our Theorem.

Proof of Theorem 7. The compactness of each $A(\omega)$ follows from Proposition 12. Also, by this Proposition and the fact that $\bigcup_{\widehat{D} \in \mathcal{D}} \Lambda(\widehat{D}, \omega) \subset A(\omega)$, we obtain

$$
\lim _{t \rightarrow+\infty} \operatorname{dist}\left(\phi\left(t, \theta_{-t} \omega, D\left(\theta_{-t} \omega\right)\right), A(\omega)\right)=0 \quad \text { for any } \quad \widehat{D} \in \mathcal{D}
$$

Now observe that, by Proposition 13,

$$
A\left(\theta_{t} \omega\right)=\overline{\bigcup_{\widehat{D} \in \mathcal{D}} \Lambda\left(\widehat{D}, \theta_{t} \omega\right)}=\overline{\bigcup_{\widehat{D} \in \mathcal{D}} \phi(t, \omega, \Lambda(\widehat{D}, \omega))}=\overline{\phi\left(t, \omega, \bigcup_{\widehat{D} \in \mathcal{D}} \Lambda(\widehat{D}, \omega)\right)} .
$$

and, as $\phi$ is continuous and $\overline{\bigcup_{\widehat{D} \in \mathcal{D}} \Lambda(\widehat{D}, \omega)}$ is compact,

$$
\overline{\phi\left(t, \omega, \bigcup_{\widehat{D} \in \mathcal{D}} \Lambda(\widehat{D}, \omega)\right)}=\phi\left(t, \omega, \overline{\bigcup_{\widehat{D} \in \mathcal{D}} \Lambda(\widehat{D}, \omega)}\right) .
$$

Consequently, we have the invariance property $\phi(t, \omega, A(\omega))=A\left(\theta_{t} \omega\right)$.

Now, let $\widehat{C} \in \mathcal{S}$ be a family such that $C(\omega)$ is closed and

$$
\lim _{t \rightarrow+\infty} \operatorname{dist}\left(\phi\left(t, \theta_{-t} \omega, B\left(\theta_{-t} \omega\right)\right), C(\omega)\right)=0
$$


Let $y$ be an element of $A(\omega)$, then $y=\lim _{n \rightarrow \infty} \phi\left(t_{n}, \theta_{-t_{n}} \omega, x_{n}\right)$,for some sequences $t_{n} \rightarrow+\infty$, $x_{n} \in B\left(\theta_{-t_{n}} \omega\right)$, and consequently $y \in \overline{C(\omega)}=C(\omega)$. Thus, $A(\omega) \subset C(\omega)$.

Remark 14 Observe that if in Theorem 7 we assume that $B(\omega)$ is closed for all $\omega \in \Omega$, and the family $\mathcal{D}$ is inclusion-closed, then it follows that the pullback $\mathcal{D}$-attractor $\widehat{A}$ belongs to $\mathcal{D}$, and hence it is the unique pullback $\mathcal{D}$-attractor which belongs to $\mathcal{D}$. This situation appears very often in applications, in particular, in our example in Section 3.

Remark 15 Note that we do not assume any structure (neither measurable nor topological) on the set of parameters $\Omega$. Consequently, this result can be applied to both nonautonomous and random dynamical systems. In particular, our concept of pullback asymptotic compactness is different from the one used by Cheban and his collaborators (see, e.g. [4]), since they require a metric structure on the space $\Omega$.

\section{Application to non-autonomous 2D-Navier-Stokes equations in unbounded domains}

Let $\mathcal{O} \subset \mathbb{R}^{2}$ be an open set, not necesarily bounded, with boundary $\partial \mathcal{O}$, and suppose that $\mathcal{O}$ satisfies the Poincaré inequality, i.e., there exists a constant $\lambda_{1}>0$ such that

$$
\lambda_{1} \int_{\mathcal{O}} \phi^{2} d x \leq \int_{\mathcal{O}}|\nabla \phi|^{2} d x \quad \text { for all } \phi \in H_{0}^{1}(\mathcal{O})
$$

Consider the following 2D-Navier-Stokes problem (for further details and notations see Lions [13] and Temam [21]):

$$
\left\{\begin{array}{l}
\frac{\partial u}{\partial t}-\nu \Delta u+\sum_{i=1}^{2} u_{i} \frac{\partial u}{\partial x_{i}}=f(t)-\nabla p \quad \text { in }(\tau,+\infty) \times \mathcal{O} \\
\operatorname{div} u=0 \text { in }(\tau,+\infty) \times \mathcal{O}, \\
u=0 \text { on }(\tau,+\infty) \times \partial \mathcal{O}, \\
u(\tau, x)=u_{0}(x), \quad x \in \mathcal{O},
\end{array}\right.
$$

To set our problem in the abstract framework, we consider the following usual abstract spaces:

$$
\mathcal{V}=\left\{u \in\left(C_{0}^{\infty}(\mathcal{O})\right)^{2} ; \operatorname{div} u=0\right\}
$$

$H=$ the closure of $\mathcal{V}$ in $\left(L^{2}(\mathcal{O})\right)^{2}$ with norm $|\cdot|$, and inner product $(\cdot, \cdot)$ where for $u, v \in$ $\left(L^{2}(\mathcal{O})\right)^{2}$,

$$
(u, v)=\sum_{j=1}^{2} \int_{\mathcal{O}} u_{j}(x) v_{j}(x) d x
$$


$V=$ the closure of $\mathcal{V}$ in $\left(H_{0}^{1}(\mathcal{O})\right)^{2}$ with norm $\|\cdot\|$, and associated scalar product $((\cdot, \cdot))$, where for $u, v \in\left(H_{0}^{1}(\mathcal{O})\right)^{2}$,

$$
((u, v))=\sum_{i, j=1}^{2} \int_{\mathcal{O}} \frac{\partial u_{j}}{\partial x_{i}} \frac{\partial v_{j}}{\partial x_{i}} d x .
$$

It follows that $V \subset H \equiv H^{\prime} \subset V^{\prime}$, where the injections are continuous and dense.

Finally, we will use $\|\cdot\|_{*}$ for the norm in $V^{\prime}$ and $\langle\cdot, \cdot\rangle$ for the duality between $V$ and $V^{\prime}$.

Consider the trilinear form $b$ on $V \times V \times V$ given by $b(u, v, w)=\sum_{i, j=1}^{2} \int_{\mathcal{O}} u_{i} \frac{\partial v_{j}}{\partial x_{i}} w_{j} d x, \quad u, v, w \in$ $V$, define $B: V \times V \rightarrow V^{\prime}$ by $\langle B(u, v), w\rangle=b(u, v, w), \quad u, v, w \in V$, and denote $B(u)=B(u, u)$.

Assume now that $u_{0} \in H, f \in L_{l o c}^{2}\left(\mathbb{R} ; V^{\prime}\right)$. For each $\tau \in \mathbb{R}$ we consider the problem:

$$
\left\{\begin{array}{l}
u \in L^{2}(\tau, T ; V) \cap L^{\infty}(\tau, T ; H) \quad \text { for all } T>\tau, \\
\frac{\mathrm{d}}{\mathrm{d} t}(u(t), v)+\nu((u(t), v))+\langle B(u(t)), v\rangle=\langle f(t), v\rangle, \quad \text { for all } v \in V, \\
\text { in the sense of scalar distributions on }(\tau,+\infty) \\
u(\tau)=u_{0} .
\end{array}\right.
$$

It follows from the results in [21] that problem (16) has a unique solution, $u\left(\cdot ; \tau, u_{0}\right)$, that moreover belongs to $C^{0}([\tau,+\infty) ; H)$.

In order to construct the non-autonomous dynamical system generated by (16), we consider $\Omega=\mathbb{R}, \theta_{t} \tau=\tau+t$ and define

$$
\phi\left(t, \tau, u_{0}\right)=u\left(t+\tau ; \tau, u_{0}\right), \quad \tau \in \mathbb{R}, \quad t \geq 0, \quad u_{0} \in H
$$

From the uniqueness of solution to problem (16), it follows that

$$
\phi\left(t+s, \tau, u_{0}\right)=\phi\left(t, s+\tau, \phi\left(s, \tau, u_{0}\right)\right), \quad \tau \in \mathbb{R}, t, s \geq 0, u_{0} \in H .
$$

Also, it is a standard task to prove that for all $\tau \in \mathbb{R}, t \geq 0$ the mapping $\phi(t, \tau, \cdot): H \rightarrow H$ defined by (17), is continuous. Consequently, the mapping $\phi$ defined by (17) is a continuous $\theta$-cocycle on $H$.

Moreover, it can be proved that $\phi$ is weakly continuous, and more exactly the following result holds true. As the proof is identical to that of Lemma 8.1 in [14] but for our particular $\phi$ given by (17), we omit it.

Proposition 16 Let $\left\{u_{0_{n}}\right\} \subset H$ be a sequence converging weakly in $H$ to an element $u_{0} \in H$. Then

$$
\phi\left(\tau, t-\tau, u_{0_{n}}\right) \rightarrow \phi\left(\tau, t-\tau, u_{0}\right), \text { weakly in } H, \text { for all } \tau \geq 0, t \in \mathbb{R}
$$




$$
\phi\left(\cdot-\tau, \tau, u_{0_{n}}\right) \rightarrow \phi\left(\cdot-\tau, \tau, u_{0}\right), \quad \text { weakly in } L^{2}(\tau, T ; V), \quad \text { for all } \tau<T \text {. }
$$

From now on, we denote $\sigma=\nu \lambda_{1}$. Let $\mathcal{R}_{\sigma}$ be the set of all functions $r: \mathbb{R} \rightarrow(0,+\infty)$ such that

$$
\lim _{t \rightarrow-\infty} e^{\sigma t} r^{2}(t)=0
$$

and denote by $\mathcal{D}_{\sigma}$ the class of all families $\widehat{D}=\{D(t) ; t \in \mathbb{R}\} \subset \mathcal{P}(H)$ such that $D(t) \subset$ $\bar{B}\left(0, r_{\widehat{D}}(t)\right)$, for some $r_{\widehat{D}} \in \mathcal{R}_{\sigma}$, where $\bar{B}\left(0, r_{\widehat{D}}(t)\right)$ denotes the closed ball in $H$ centered at zero with radius $r_{\widehat{D}}(t)$.

Now, we can prove the following result:

Theorem 17 Suppose that $f \in L_{\text {loc }}^{2}\left(\mathbb{R} ; V^{\prime}\right)$ is such that

$$
\int_{-\infty}^{t} e^{\sigma \xi}\|f(\xi)\|_{*}^{2} d \xi<+\infty \quad \text { for all } t \in \mathbb{R}
$$

Then, there exists a unique global pullback $\mathcal{D}_{\sigma}$-attractor belonging to $\mathcal{D}_{\sigma}$ for the cocycle $\phi$ defined by (17).

Proof.- Let $t \in \mathbb{R}, \tau \geq 0$ and $u_{0} \in H$ be fixed, and denote

$$
u(r)=u\left(r ; t-\tau, u_{0}\right)=\phi\left(r-t+\tau, t-\tau, u_{0}\right), \text { for } r \geq t-\tau
$$

Taking into account that $b(u, v, v)=0$, it follows

$$
\frac{\mathrm{d}}{\mathrm{d} r}\left(e^{\sigma r}|u(r)|^{2}\right)+2 \nu e^{\sigma r}\|u(r)\|^{2}=\sigma e^{\sigma r}|u(r)|^{2}+2 e^{\sigma r}\langle f(r), u(r)\rangle,
$$

in $\left(C_{0}^{\infty}(t-\tau,+\infty)\right)^{\prime}$, and consequently, by (15),

$$
e^{\sigma t}|u(t)|^{2} \leq e^{\sigma(t-\tau)}\left|u_{0}\right|^{2}+\frac{1}{\nu} \int_{t-\tau}^{t} e^{\sigma \xi}\|f(\xi)\|_{*}^{2} d \xi \quad \text { for all } \tau \geq 0 .
$$

Let $\widehat{D} \in \mathcal{D}_{\sigma}$ be given. From (24), we easily obtain

$$
\left|\phi\left(\tau, t-\tau, u_{0}\right)\right|^{2} \leq e^{-\sigma \tau} r_{\widehat{D}}^{2}(t-\tau)+\frac{e^{-\sigma t}}{\nu} \int_{-\infty}^{t} e^{\sigma \xi}\|f(\xi)\|_{*}^{2} d \xi,
$$

for all $u_{0} \in D(t-\tau), t \in \mathbb{R}, \tau \geq 0$.

Denote by $R_{\sigma}(t)$ the nonnegative number given for each $t \in \mathbb{R}$ by

$$
\left(R_{\sigma}(t)\right)^{2}=\frac{2 e^{-\sigma t}}{\nu} \int_{-\infty}^{t} e^{\sigma \xi}\|f(\xi)\|_{*}^{2} d \xi
$$

and consider the family $\widehat{B}_{\sigma}$ of closed balls in $H$ defined by

$$
B_{\sigma}(t)=\left\{v \in H ;|v| \leq R_{\sigma}(t)\right\} .
$$


It is straightforward to check that $\widehat{B}_{\sigma} \in \mathcal{D}_{\sigma}$, and moreover, by (21) and (25), the family $\widehat{B}_{\sigma}$ is pullback $\mathcal{D}_{\sigma}$-absorbing for the cocycle $\phi$.

According to Theorem 7 and Remark 14, to finish the proof of the theorem we only have to prove that $\phi$ is pullback $\mathcal{D}_{\sigma}$-asymptotically compact.

Let us fix $\widehat{D} \in \mathcal{D}_{\sigma}, t \in \mathbb{R}$, a sequence $\tau_{n} \rightarrow \infty$, and a sequence $u_{0_{n}} \in D\left(t-\tau_{n}\right)$. We must prove that from the sequence $\left\{\phi\left(\tau_{n}, t-\tau_{n}, u_{0_{n}}\right)\right\}$ we can extract a subsequence that converges in $H$.

As the family $\widehat{B}_{\sigma}$ is pullback $\mathcal{D}_{\sigma}$-absorbing, for each integer $k \geq 0$ there exists a $\tau_{\widehat{D}}(k) \geq 0$ such that

$$
\phi(\tau, t-\tau-k, D(t-\tau-k)) \subset B_{\sigma}(t-k) \quad \text { for all } \tau \geq \tau_{\widehat{D}}(k) .
$$

Observe now that for $\tau \geq \tau_{\widehat{D}}(k)+k$ it follows from (28) that

$$
\phi(\tau-k, t-\tau, D(t-\tau)) \subset B_{\sigma}(t-k) .
$$

It is not difficult to conclude from (29), by a diagonal procedure, the existence of a subsequence $\left\{\left(\tau_{n^{\prime}}, u_{0_{n^{\prime}}}\right)\right\} \subset\left\{\left(\tau_{n}, u_{0_{n}}\right)\right\}$, and a sequence $\left\{w_{k} ; k \geq 0\right\} \subset H$, such that for all $k \geq 0$ and $w_{k} \in B_{\sigma}(t-k)$,

$$
\phi\left(\tau_{n^{\prime}}-k, t-\tau_{n^{\prime}}, u_{0_{n^{\prime}}}\right) \rightarrow w_{k} \quad \text { weakly in } H \text {. }
$$

Observe that, by Proposition 16,

$$
\begin{aligned}
w_{0} & =\text { weak }-\lim _{n^{\prime} \rightarrow \infty} \phi\left(\tau_{n^{\prime}}, t-\tau_{n^{\prime}}, u_{0_{n^{\prime}}}\right) \\
& =\text { weak }-\lim _{n^{\prime} \rightarrow \infty} \phi\left(k, t-k, \phi\left(\tau_{n^{\prime}}-k, t-\tau_{n^{\prime}}, u_{0_{n^{\prime}}}\right)\right) \\
& =\phi\left(k, t-k,\left(\text { weak }-\lim _{n^{\prime} \rightarrow \infty} \phi\left(\tau_{n^{\prime}}-k, t-\tau_{n^{\prime}}, u_{0_{n^{\prime}}}\right)\right)\right),
\end{aligned}
$$

i.e.,

$$
\phi\left(k, t-k, w_{k}\right)=w_{0}, \quad \text { for all } k \geq 0 .
$$

Then, by the lower semi-continuity of the norm,

$$
\left|w_{0}\right| \leq \liminf _{n^{\prime} \rightarrow \infty}\left|\phi\left(\tau_{n^{\prime}}, t-\tau_{n^{\prime}}, u_{0_{n^{\prime}}}\right)\right| .
$$

If we now prove that also

$$
\limsup _{n^{\prime} \rightarrow \infty}\left|\phi\left(\tau_{n^{\prime}}, t-\tau_{n^{\prime}}, u_{0_{n^{\prime}}}\right)\right| \leq\left|w_{0}\right|
$$

then we will have

$$
\lim _{n^{\prime} \rightarrow \infty}\left|\phi\left(\tau_{n^{\prime}}, t-\tau_{n^{\prime}}, u_{0_{n^{\prime}}}\right)\right|=\left|w_{0}\right|,
$$

and this, together with the weak convergence, will imply the strong convergence in $H$ of $\phi\left(\tau_{n^{\prime}}, t-\tau_{n^{\prime}}, u_{0_{n^{\prime}}}\right)$ to $w_{0}$. 
In order to prove (33), we consider the Hilbert norm in $V$ given by

$$
[u]^{2}=\nu\|u\|^{2}-\frac{\sigma}{2}|u|^{2},
$$

which by (15) is equivalent to the norm $\|u\|$ in $V$.

Then, it is immediate that for all $t \in \mathbb{R}, \tau \geq 0$ and all $u_{0} \in H$,

$$
\begin{aligned}
& \left|\phi\left(\tau, t-\tau, u_{0}\right)\right|^{2}=\left|u_{0}\right|^{2} e^{-\sigma \tau} \\
& +2 \int_{t-\tau}^{t} e^{\sigma(\xi-t)}\left(\left\langle f(\xi), \phi\left(\xi-t+\tau, t-\tau, u_{0}\right)\right\rangle-\left[\phi\left(\xi-t+\tau, t-\tau, u_{0}\right)\right]^{2}\right) d \xi
\end{aligned}
$$

and, thus, for all $k \geq 0$ and all $\tau_{n^{\prime}} \geq k$,

$$
\begin{aligned}
& \left|\phi\left(\tau_{n^{\prime}}, t-\tau_{n^{\prime}}, u_{0_{n^{\prime}}}\right)\right|^{2}=\left|\phi\left(k, t-k, \phi\left(\tau_{n^{\prime}}-k, t-\tau_{n^{\prime}}, u_{0_{n^{\prime}}}\right)\right)\right|^{2} \\
& =e^{-\sigma k}\left|\phi\left(\tau_{n^{\prime}}-k, t-\tau_{n^{\prime}}, u_{0_{n^{\prime}}}\right)\right|^{2} \\
& +2 \int_{t-k}^{t} e^{\sigma(\xi-t)}\left\langle f(\xi), \phi\left(\xi-t+k, t-k, \phi\left(\tau_{n^{\prime}}-k, t-\tau_{n^{\prime}}, u_{0_{n^{\prime}}}\right)\right)\right\rangle d \xi \\
& -2 \int_{t-k}^{t} e^{\sigma(\xi-t)}\left[\phi\left(\xi-t+k, t-k, \phi\left(\tau_{n^{\prime}}-k, t-\tau_{n^{\prime}}, u_{0_{n^{\prime}}}\right)\right)\right]^{2} d \xi .
\end{aligned}
$$

As by (29),

$$
\phi\left(\tau_{n^{\prime}}-k, t-\tau_{n^{\prime}}, u_{0_{n^{\prime}}}\right) \in B_{\sigma}(t-k) \quad \text { for all } \tau_{n^{\prime}} \geq \tau_{\widehat{D}}(k)+k, k \geq 0,
$$

we have

$$
\limsup _{n^{\prime} \rightarrow \infty}\left(e^{-\sigma k}\left|\phi\left(\tau_{n^{\prime}}-k, t-\tau_{n^{\prime}}, u_{0_{n^{\prime}}}\right)\right|^{2}\right) \leq e^{-\sigma k} R_{\sigma}^{2}(t-k) \quad k \geq 0 .
$$

On the other hand, as $\phi\left(\tau_{n^{\prime}}-k, t-\tau_{n^{\prime}}, u_{0_{n^{\prime}}}\right) \rightarrow w_{k}$ weakly in $H$, by Proposition 16 we have

$$
\phi\left(\cdot-t+k, t-k, \phi\left(\tau_{n^{\prime}}-k, t-\tau_{n^{\prime}}, u_{0_{n^{\prime}}}\right)\right) \rightarrow \phi\left(\cdot-t+k, t-k, w_{k}\right),
$$

weakly in $L^{2}(t-k, t ; V)$.

Taking into account that, in particular, $e^{\sigma(\xi-t)} f(\xi) \in L^{2}\left(t-k, t ; V^{\prime}\right)$, we obtain from (37),

$$
\begin{aligned}
& \lim _{n^{\prime} \rightarrow \infty} \int_{t-k}^{t} e^{\sigma(\xi-t)}\left\langle f(\xi), \phi\left(\xi-t+k, t-k, \phi\left(\tau_{n^{\prime}}-k, t-\tau_{n^{\prime}}, u_{0_{n^{\prime}}}\right)\right\rangle d \xi\right. \\
& =\int_{t-k}^{t} e^{\sigma(\xi-t)}\left\langle f(\xi), \phi\left(\xi-t+k, t-k, w_{k}\right)\right\rangle d \xi .
\end{aligned}
$$

Moreover, as $\left(\int_{t-k}^{t} e^{\sigma(\xi-t)}[v(\xi)]^{2} d \xi\right)^{1 / 2}$ defines a norm in $L^{2}(t-k, t ; V)$ which is equivalent to the usual one, we also obtain from (37),

$$
\begin{aligned}
& \int_{t-k}^{t} e^{\sigma(\xi-t)}\left[\phi\left(\xi-t+k, t-k, w_{k}\right)\right]^{2} d \xi \\
& \leq \liminf _{n^{\prime} \rightarrow \infty} \int_{t-k}^{t} e^{\sigma(\xi-t)}\left[\phi\left(\xi-t+k, t-k, \phi\left(\tau_{n^{\prime}}-k, t-\tau_{n^{\prime}}, u_{0_{n^{\prime}}}\right)\right)\right]^{2} d \xi .
\end{aligned}
$$


Then, from (35), (36), (38) and (39), we easily obtain

$$
\begin{aligned}
& \limsup _{n^{\prime} \rightarrow \infty}\left|\phi\left(\tau_{n^{\prime}}, t-\tau_{n^{\prime}}, u_{0_{n^{\prime}}}\right)\right|^{2} \leq e^{-\sigma k} R_{\sigma}^{2}(t-k) \\
& +2 \int_{t-k}^{t} e^{\sigma(\xi-t)}\left(\left\langle f(\xi), \phi\left(\xi-t+k, t-k, w_{k}\right)\right\rangle-\left[\phi\left(\xi-t+k, t-k, w_{k}\right)\right]^{2}\right) d \xi .
\end{aligned}
$$

Now, from (31) and (34),

$$
\begin{aligned}
& \left|w_{0}\right|^{2}=\left|\phi\left(k, t-k, w_{k}\right)\right|^{2}=\left|w_{k}\right|^{2} e^{-\sigma k} \\
& +2 \int_{t-k}^{t} e^{\sigma(\xi-t)}\left(\left\langle f(\xi), \phi\left(\xi-t+k, t-k, w_{k}\right)\right\rangle-\left[\phi\left(\xi-t+k, t-k, w_{k}\right)\right]^{2}\right) d \xi
\end{aligned}
$$

From (40) and (41), we have

$$
\begin{aligned}
\limsup _{n^{\prime} \rightarrow \infty}\left|\phi\left(\tau_{n^{\prime}}, t-\tau_{n^{\prime}}, u_{0_{n^{\prime}}}\right)\right|^{2} & \leq e^{-\sigma k} R_{\sigma}^{2}(t-k)+\left|w_{0}\right|^{2}-\left|w_{k}\right|^{2} e^{-\sigma k} \\
& \leq e^{-\sigma k} R_{\sigma}^{2}(t-k)+\left|w_{0}\right|^{2}
\end{aligned}
$$

and thus, taking into account that

$$
e^{-\sigma k} R_{\sigma}^{2}(t-k)=\frac{2 e^{-\sigma t}}{\nu} \int_{-\infty}^{t-k} e^{\sigma \xi}\|f(\xi)\|_{*}^{2} d \xi \rightarrow 0
$$

when $k \rightarrow+\infty$, we easily obtain (33) from the last inequality.

\section{Conclusions and final remarks}

We have introduced the concept of pullback asymptotic compactness and proved the existence of a minimal pullback attractor under very general conditions. In fact, this pullback asymptotic compactness and the existence of a family of absorbing sets ensure that pullback attractors exist. Although in the framework of random (and non-autonomous) dynamical systems one often imposes (even in the compact case) that the attractor belongs to the universe of attracted sets, it is not necessary in our analysis and, in this sense, our assumptions are much more general. Despite the fact that we cannot prove the uniqueness of the pullback attractor under our general assumptions, we are able to prove that our pullback attractor is minimal.

Needless to say that our theory could be applied to other interesting models, but we only considered one of them to show how it works.

There are some other interesting problems to be treated as, for example, the finitedimensionality of the pullback attractor, but this will be the topic of another paper.

Acknowledgement. We would like to thank the referee for helpful comments and suggestions. We also express our thanks to Pedro Marín-Rubio for his interesting comments on the topic of this paper. 


\section{References}

[1] T. Caraballo \& J. Real, Attractors for 2D-Navier-Stokes models with delays, J. Diff. Eqns. 205(2004), 270-296.

[2] T. Caraballo, P.E. Kloeden \& J. Real, Pullback and forward attractors for a damped wave equation with delays, Stochastics $\&$ Dynamics 4(3) (2004), 405-423.

[3] T. Caraballo \& J.A. Langa, On the upper semicontinuity of cocycle attractors for nonautonomous and random dynamical systems, Dynamics of Continuous Discrete and Impulsive Systems A, 10(2003), 491-514.

[4] D. Cheban \& J. Duan, Almost periodic solutions and global attractors of non-autonomous Navier-Stokes equations, Journal of Dynamics and Differential Equations, 16(2004), 1-33.

[5] V.V. Chepyzhov \& M.I. Vishik, Attractors for Equations of Mathematical Physics, Colloquium publications fol. 49, American Mathematical Society, Providence, Rhode Island, (2002).

[6] J.W. Cholewa \& T. Dlotko, Global attractors in abstract parabolic problems, London Mathematical Society Lecture Note Series, 278. Cambridge University Press, Cambridge, $(2000)$

[7] I.D. Chueshov, Introduction to the Theory of Infinite-Dimensional Dissipative Systems, Acta Scientific Publishing House, Kharkiv, (2002).

[8] I.D. Chueshov, Monotone Random Systems- Theory and Applications, Lecture Notes in Mathematics, vol. 1779, Springer, Berlin (2002).

[9] H. Crauel, A. Debussche \& F. Flandoli, Random attractors, J. Dyn. Diff. Eq. 9(1995), No. $2,307-341$.

[10] F. Flandoli \& B. Schmalfuss, Random attractors for the 3D stochastic Navier-Stokes equation with multiplicative white noise, Stochastics and Stochastics Reports 59(1996), 2145.

[11] P.E. Kloeden \& B. Schmalfuss, Asymptotic behaviour of nonautonomous difference inclusions. Systems Control Lett. 33(1998), no. 4, 275-280.

[12] J.A. Langa \& B. Schmalfuss, Finite dimensionality of attractors for non-autonomous dynamical systems given by partial differential equations, Stochastics and Dynamics $4(2004)$, no. $3,385-404$.

[13] J.L. Lions, Quelques méthodes de résolution des problèmes aux limites non linéaires, Paris; Dunod, Gauthier-Villars, (1969).

[14] G. Łukaszewicz \& W. Sadowski, Uniform attractor for 2D magneto-micropolar fluid flow in some unbounded domains, Z. angew. Math. Phys. 55(2004), 1-11.

[15] R. K. Miller, Almost periodic differential equations as dynamical systems with applications to the existence of almost periodic solutions, J. Diff. Eqns. 1(1965), 337-395. 
[16] I. Moise, R. Rosa \& X. Wang, Attractors for noncompact nonautonomous systems via energy equations, Discrete and Continuous Dynamical Systems 10(2004), No. 1 \& 2, 473-496.

[17] J. Robinson, Infinite-Dimensional Dynamical Systems, Cambridge University Press, Cambridge, (2001).

[18] R. Rosa, The global attractor for the $2 D$ Navier-Stokes flow on some unbounded domains, Nonlinear Analysis, TMA 32(1998), No. 1, 71-85.

[19] B. Schmalfuss, Attractors for non-autonomous dynamical systems, in Proc. Equadiff 99, Berlin, Eds. B. Fiedler, K. Gröger and J. Sprekels (World Scientific, 2000), pp. 684-689.

[20] G.R. Sell, Non-autonomous differential equations and topological dynamics, I, II Trans. Amer. Math. Soc. 127(1967), 241-262, 263-283.

[21] R. Temam, Navier-Stokes equations, Theory and Numerical Analysis, 2nd. ed., North Holland, Amsterdam, (1979).

[22] R. Temam, Infinite Dimensional Dynamical Systems in Mechanics and Physics, SpringerVerlag, New York, (1988).

[23] M.I. Vishik, Asymptotic behaviour of solutions of evolutionary equations, Cambridge Univ. Press, Cambridge, (1992). 